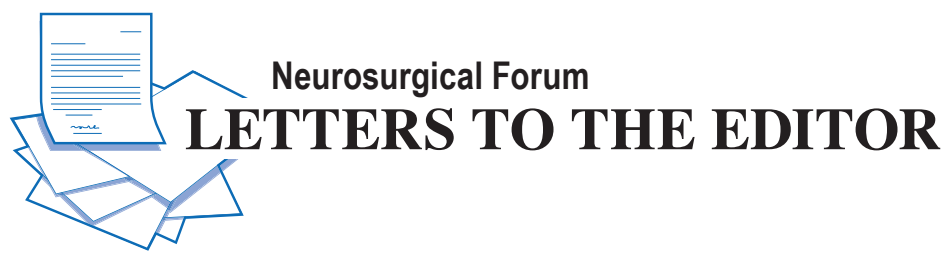

\section{Endoscopic ultrasonic aspirator for the removal of intraventricular tumors}

TO THE EDITOR: We read with the greatest of interest the article by Cinalli et al. ${ }^{1}$ in which the authors summarize their experience with the Söring endoscopic ultrasonic aspirator for the removal of intraventricular tumors (Cinalli G, Imperato A, Mirone G, et al: Initial experience with endoscopic ultrasonic aspirator in purely neuroendoscopic removal of intraventricular tumors. J Neurosurg Pediatr 19:325-332, March 2017). It is an excellent account describing the use of the aspirator in 12 children with intra/paraventricular and suprasellar/third ventricular lesions. We congratulate the authors as we believe that the advancement of neuroendoscopy will only be possible with the help of such reports. In this spirit we briefly share our experience using the same device, with the hope of expanding its use and overcoming some of its limitations.

We had previously recounted our use of the aspirator to remove a choroid plexus papilloma in an infant ${ }^{2}$ and we have since used the device in a total of 19 patients (pediatric and adult) and 22 procedures (intra/paraventricular lesions as well as sellar/suprasellar ones extending into or towards the third ventricle).

Although the Söring endoscopic handpiece was built for the sole use with the Gaab scope, we modified it to allow use with other endoscopes. Due to its large oval working channel, our preferred endoscope for tumors is the Aesculap InVent, and it is this scope that we use most commonly with the endoscopic aspirator. When using the aspirator, we found that minimal suction was required but we often required high cavitating power. We use copious wash under pressure to ensure that the ventricles/cavities are kept expanded. Pressured wash is safe using endoscopes with large channels, but extreme care should be exercised with smaller channels. We found the ability to sweep from side to side and angulate the aspirator within the large oval working channel most useful. The inability to do this was a limiting factor in Dr. Cinalli's experience. We found soft tumors and cystic lesions easiest to address and harder or larger ones more difficult. There was no morbidity or mortality directly or indirectly related to our use of the aspirator.
We agree with Cinalli et al. that the Söring endoscopic ultrasonic aspirator is safe and reliable. We echo their opinion that this tool has the potential to expand the spectrum of intracranial pathologies treatable by modern neuroendoscopic means, but not if it continues to be manufactured with only one endoscope in mind and not without further development. This development must not be driven by companies/product developers/engineers alone with neurosurgeons only involved at the end stage; it must be guided, from the outset, by the needs and ideas of the surgeons using it. Ensuring this happens is, of course, no easy feat, but perhaps with the assistance of the International Federation of Neuroendoscopy, it may become possible.

Bassel Zebian, MBBS, BSc, MRCS(Eng), FRCS(NeuroSurg) Nida Kalyal, BSc, MBBS, AKC Charlotte Burford, MSc, BA John Hanrahan, BSc Ali Ansaripour Wisam AI-Faiadh, MBBS, MSc King's College Hospital, London, United Kingdom

\section{References}

1. Cinalli G, Imperato A, Mirone G, Di Martino G, Nicosia $\mathrm{G}$, Ruggiero C, et al: Initial experience with endoscopic ultrasonic aspirator in purely neuroendoscopic removal of intraventricular tumors. J Neurosurg Pediatr 19:325-332, 2017

2. Gerard AWL, Tailor J, Gradil C, Thakur B, Zebian B: Endoscopic resection of intraventricular choroid plexus papillomas in infants. J Neurosurg Pediatr 19:122-125, 2017 (Letter)

\section{Disclosures}

The authors report no conflict of interest.

\section{Correspondence}

Bassel Zebian: bzebian@gmail.com.

INCLUDE WHEN CITING

Published online May 25, 2018; DOI: 10.3171/2018.1.PEDS1811.

\section{Response}

We are grateful to Dr. Zebian and his colleagues for sharing their experience with us in the neuroendoscopic 
removal of intraventricular tumors. For many years this surgery was performed by pioneering neuroendoscopists with limited instruments that could easily be defined as rudimentary if compared with the technological armamentarium usually available to microsurgeons. Small tumors were removed piecemeal using only coagulation and small forceps, and this long work always resulted in rapidly blurred vision due to CSF contamination from blood oozing from the tumor. Reviewing old videos of that kind of surgery, I found not infrequently that more than $70 \%$ of the video was spent on irrigation to recover acceptable vision and only approximately $30 \%$ was dedicated to actual surgical maneuvers. Results were good, but indications were limited to small tumors $(<1.5 \mathrm{~cm})$ and conversion to microsurgery was possible, but extremely rare. The endoscopic ultrasonic aspirator was, in our practice, a real breakthrough. A tumor cyst could be aspirated and emptied in a few seconds without any spillage of cystic fluid within the ventricles while maintaining perfect visibility in clear CSF at the beginning of surgery. Intraventricular tumors of larger size (up to $4 \mathrm{~cm}$ ) could be removed in a shorter time with a safer technique due to better vision quality. Aspiration through the aspirating cannula allows one to aspirate the blood oozing from the tumor and continuous irrigation guarantees a clear microenvironment at the tip of the endoscope that warrants good or excellent visibility in the field of work.

In addition to this, the dry field technique, firstly described by $\mathrm{Gaab}^{1}$ and excellently used by Dr. Zebian in his report, ${ }^{2}$ allows one to easily, safely, and rapidly manage difficult bleeding situations that only a few years ago would have caused a surgeon to abort the procedure and possibly convert it to a standard microsurgical procedure.
Dr. Zebian also proposed a modification of the long cannula of the ultrasonic aspirator: decreasing its outer diameter allows its use in endoscopes other than the Gaab, with the possibility to angle it within the working channel, thus enhancing surgical maneuverability. We perform these procedures using a Gaab endoscope, which is the one for which the ultrasonic aspirator was designed, so we do not need adaptation, but the idea of having the possibility to tilt and angle the cannula within the working channel without moving the endoscope is certainly intriguing. I congratulate Dr. Zebian for the nice case series and results that he was able to describe in such a short period of time, and I join him in the hope of a better and more intensive cooperation with engineers during the project phase. This is by far the only way to rapidly improve surgical instrumentation.

Giuseppe Cinalli, MD

Santobono-Pausilipon Children's Hospital, Naples, Italy

\section{References}

1. Gaab MR: Neuroendoscopic technology, in Torres-Corzo JG, Rangel-Castilla L, Nakaji P (eds): Neuroendoscopic Surgery. New York: Thieme, 2016, pp 11-23

2. Gerard AWL, Tailor J, Gradil C, Thakur B, Zebian B: Endoscopic resection of intraventricular choroid plexus papillomas in infants. J Neurosurg Pediatr 19:122-125, 2017 (Letter)

INCLUDE WHEN CITING

Published online May 25, 2018; DOI: 10.3171/2018.2.PEDS1867.

CAANS 2018, except where prohibited by US copyright law 